becoming increasingly interested in crystal dynamics it would have been advantageous to both sides if the relationship between the Schoenflies and Hermann-Mauguin symbolism had been made clear, the translation-containing symmetry elements of space groups described, and a discussion given of the derivation of the isomorphous point group from the symmetry elements of the crystallographic space group. However, this would have been mainly an educational gain, as for most particular examples the necessary relationships are to be found in the comprehensive tables.

The high price of this small book may be the result of the extended tabulated matter it contains. However, it is a very useful handbook which should certainly be on the bookshelves of all interested in the vibrational spectra of crystals, and a number of other spectroscopists besides.

N. Sheppard

\section{Denaturation Dangers}

Certainty and Uncertainty in Biochemical Techniques. By Harold Hillman. Pp. $\mathrm{x}+126$. (Surrey University: Henley-on-Thames, October 1972.) $£ 2.85$.

EVERY argument used in science has its loopholes, and some of the most dangerous are those easily forgotten because the arguments have ceased to be stated explicitly because they refer to popular techniques. Hence a book that aims to scrutinize the assumptions of such techniques is welcome. In biology care must be taken to compare results at different levels of study, and the danger in the more controlled situations of the biochemist is that they are "unphysiological", frequently because the experimenter may alter his material in ways of which he is not aware. The author examines the six techniques of subcellular fractionation, histochemistry, electron microscopy, measurements of radioactivity, electrophoresis and chromatography. After this he makes some general comments and summarizes his conclusions, largely that "an experiment is only as good as its controls".

Although several of the real difficulties of using and interpreting each selected technique are given, and several wise comments are spread throughout the book, its overall approach is largely spoilt by the author's concentration on heat. Because heating can harm biological material, each experimental situation is assessed largely in terms of the heating that occurs. Even that produced by impact of subcellular particles on the bottom of the centrifuge tube is reckoned one of the dangers of subcellular fractionation.

Of course, the author is right that a protein may be denatured by heat during its extraction and that a procedure that keeps it cool diminishes this risk. But much can be done to assess whether the changes feared have occurred, by examining the homogeneity of the product and by comparing proteins extracted in different ways. This will not provide a complete answer, for a "denaturation" could involve the quantitative conversion of a protein into a single altered form. In the book such tests are little discussed and the reader is left with repeated warnings of the dangers of local heating and the difficulties of assessing its degree.

Electrophoresis seems to be a case where the author's concentration would be justified because most of the difficulty of scaling it up is the dissipation of heat. The rate of separation depends on the potential gradient, and the conductivity of the medium must be kept high because otherwise the substances being separated would significantly affect this conductivity. The potential gradient would then differ between the centre and edges of each band, and this would result in distortion and spread of each peak. But the author does not consider this, and discusses electrophoresis in terms of the differential heating of different bands. The controls of checking that the mobility remains constant, both with time and with potential gradient up to the highest used, are not discussed, let alone re-running of the fractions. I am not trying to deny the danger of overheating; indeed I have myself turned up the potential too high and seen a spot of haemoglobin that had been running nicely stop dead as it denatured. But here, as throughout, more emphasis on the possible checks, and less repetition of how unknown and dangerous heating effects are, would have given the book greater balance and value.

H. B. F. Dixon

\section{Engineering in Context}

Creativity and Innovation in Engineering. Edited by S. A. Gregory. Pp. 313. (Butterworth: London, 1972.) £5.

ANY word in the engineer's vocabulary that is associated with design seems doomed to ambiguity. For example, if you find the words creativity or inno. vation on the cover of a book you cannot tell what they are intended to mean until you look inside. Chameleon like, they take their colour from the leaves in which they are hidden. There is a whole spectrum of possibilities. At one extreme you find the enthusiastic designer regarding creative work only meaningly portrayed in the work that has been created, that is, the final structure or machine. To him the elegance of the design and its embodied mathematics is the test of creativity. His book would be full of diagrams and calculations, for drawings would be his prose, mathematics his grammar, and differential equations his poetry. At the other extreme would be a book totally concerned with the context of designing, that is, the educational background, the commercial foreground and environmental constraints within which the designer must design. The spectrum ranges from pure content to pure context.

If you find it difficult to imagine a book on "creativity and innovation in engineering" which is totally concerned with context and never shows or discusses a design at all, you can overcome your difficulty by buying one, for this is the title used by S. A. Gregory's book. It is a collection of papers prepared by the Design and Innovation Group of the University of Aston, Birmingham, under the joint sponsorship of the Working Party on Creativity of the Council Engineering Institution and of the Design Research Society.

Compressed into it are eighteen papers dealing with various aspects of the subject. Inevitably, perhaps, there is a good deal of overlap, but, surprisingly, there are noticeable gaps. For example, we are told on page 81 that "University education in Britain was until lately for the social elite and did not concern itself with the instruction and development of practical engineers at all". To many, like myself, who have been designing for many years by utilizing the realistic principles that we learnt in the Engineering Department at Cambridge, it is a little surprising to learn that, since 1783, the University has solely concentrated on training impractical engineers. Actually students at Cambridge have been required for many years not only to innovate new designs but each of them has his design costed, built and tested, and an account of this and the lectures on creativity would have added much needed substance to the section on education.

Although this book contains a useful summary of theories about the context of design, many of the chapters seem to finish before they have finished. Concluding is common, but conclusions are much rarer. Perhaps the best part of the book is the emphasis on the necessity of industry to exploit innovation and encourage an atmosphere that will breed it, but its general effect is to give the impression that it is easier to break the subject up into little bits than to put it together again to present a unified and persuasive picture.

G. L. GlegG 